\title{
Long-Term Microgravity Effects on Isometric Handgrip and Precision Pinch Force with Visual and Proprioceptive Feedback
}

\author{
Ilario Puglia, Michele Balsamo, Marco Vukich, and Valfredo Zolesi $\mathbb{D}$ \\ Kayser Italia Srl, Via di Popogna 501, 57128 Livorno, Italy \\ Correspondence should be addressed to Valfredo Zolesi; v.zolesi@kayser.it
}

Received 27 April 2017; Accepted 20 July 2017; Published 4 March 2018

Academic Editor: Satoshi Matsumoto

Copyright (C) 2018 Ilario Puglia et al. This is an open access article distributed under the Creative Commons Attribution License, which permits unrestricted use, distribution, and reproduction in any medium, provided the original work is properly cited.

\begin{abstract}
The study and analysis of human physiology during short- and long-duration space flights are the most valuable approach in order to evaluate the effect of microgravity on the human body and to develop possible countermeasures in prevision of future exploratory missions and Mars expeditions. Hand performances such as force output and manipulation capacity are fundamental for astronauts' intra- and extravehicular activities. Previous studies on upper limb conducted on astronauts during short-term missions (10 days) indicated a temporary partial reduction in the handgrip maximum voluntary contraction (MVC) followed by a prompt recovery and adaptation to weightlessness during the last days of the mission. In the present study, we report on the "Crew's Health: Investigation on Reduced Operability" (CHIRO) protocol, developed for handgrip and pinch force investigations, performed during the six months increment 7 and increment 8 (2003-2004) onboard International Space Station (ISS). We found that handgrip and pinch force performance are reduced during long-term increments in space and are not followed by adaptation during the mission, as conversely reported during short-term increment experiments. The application of protocols developed in space will be eligible to astronauts during long-term space missions and to patients affected by muscle atrophy diseases or nervous system injury on Earth.
\end{abstract}

\section{Introduction}

The microgravity environment alters the musculoskeletal system during short- and long-duration missions, resulting in atrophy of bone and muscle tissues [1]. It is therefore essential for space mission success to apply effective countermeasures such as physical exercise and tailored diets [2] to mitigate such effects during short- and long-term missions. Astronauts strongly rely on the use of their upper limbs to control their movements and to operate and perform tasks onboard or outside ISS during extravehicular activities (EVAs). During EVAs, the upper limbs and specially the hands experience fatigue; this could pose a critical risk for the operations to be performed and for the overall mission success. Therefore, several technological advancements have been proposed to the astronauts' spacesuit and in particular the pressurized suit glove in order to reduce hand fatigue $[3,4]$. Hence, understanding how the upper limb responds during prolonged time in microgravity is fundamental in order to advance knowledge for the establishment of preventive or rehabilitation protocols for space crews involved in long-lasting missions.

In order to support investigations on astronauts' upper limb performance during a space mission, a dedicated facility, the Hand Posture Analyzer (HPA) [5, 6] (Figure 1), was designed, developed, and manufactured by Kayser Italia under an Italian Space Agency (ASI) contract. A reduced set of instruments named handgrip dynamometer (HGD) was produced under a European Space Agency (ESA) contract in the frame of the ESA-NASA barter agreement. The HPA returned on Earth on July 21, 2011, onboard the last Shuttle mission. The HPA in its preliminary version took part in the Marco Polo Mission (Soyuz TM34, April 25 to May 3, 2002) [5] and consisted of a handgrip dynamometer (HGD, Figure 1(a)), a pinch force dynamometer (PFD, Figure 1(b)), and a PC with data acquisition system and software (SW). The findings of the Marco Polo Mission, that is the fact that the maximum voluntary contraction (MVC) was decreasing during the first period followed by recovery in the last days was confirmed by the same astronauts during 


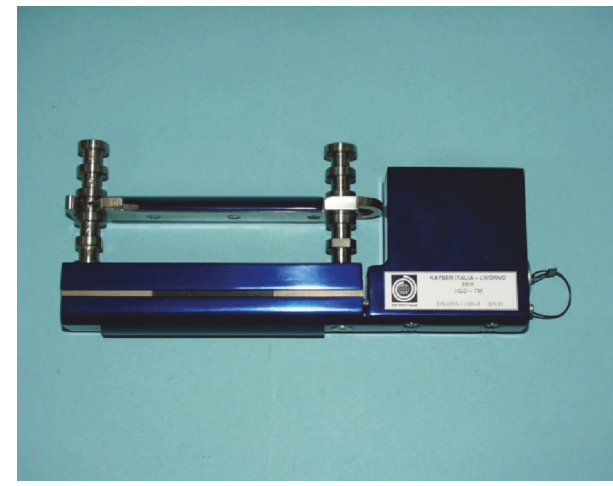

(a)

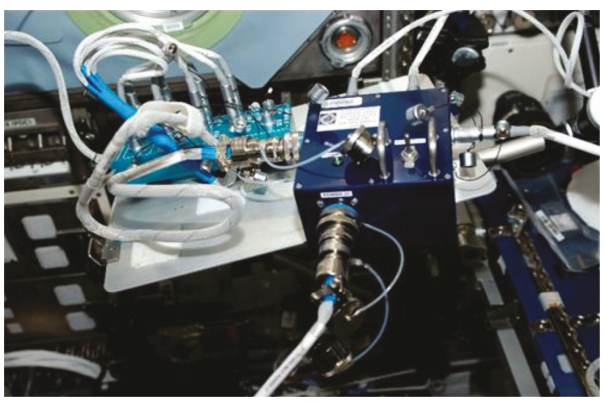

(c)

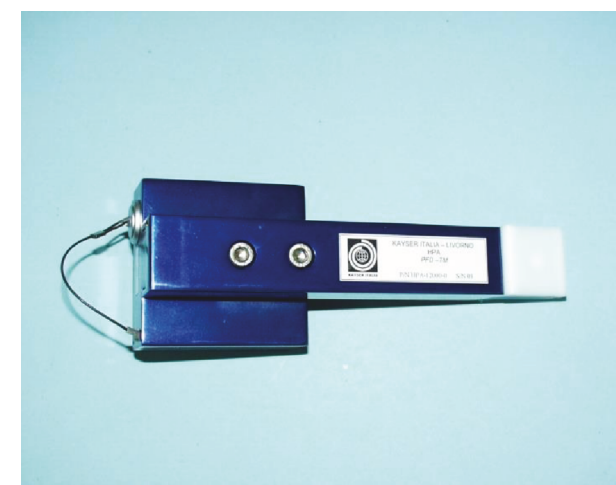

(b)

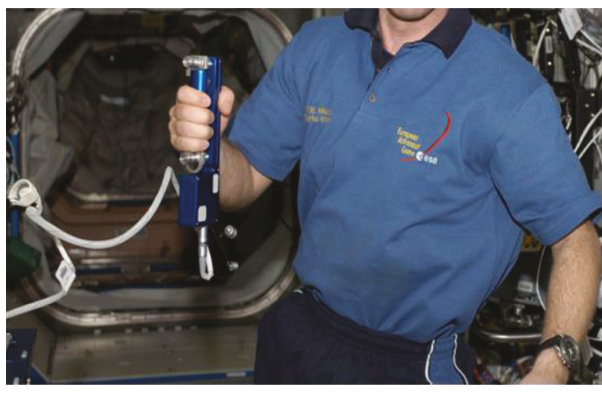

(d)

FIgure 1: CHIRO instrumentation. (a) Handgrip dynamometer (HGD) tool. (b) Pinch force dynamometer (PFD) tool. (c) Interface box (IBOX) onboard ISS. (d) HPA used by an astronaut onboard ISS during the mission.

the Eneide Mission (Soyuz 10S, April 15 to April 24, 2005) and then during the Esperia Mission (STS 120, October 23 to November 7, 2007, our unpublished data). The observed astronauts' MVC behavior resembled the one shown by people affected by the sopite syndrome, a neurological disorder triggered by prolonged periods of motion that relates to symptoms of drowsiness, fatigue, and mood changes [7].

To gain insight into how the control of a handgrip and a precision lateral pinch force is influenced by reduced gravity and to quantify the adaptive normalization along missions, two astronauts periodically executed the CHIRO protocol with HPA in 2003 and 2004, during long-term increments 7 and 8 (an increment on the ISS is the period of permanence of the crew onboard, lasting typically 6 months). In both missions, the analyses were focused on understanding the differences between short-term and long-term exposure to weightlessness and between ground and flight sessions. During the CHIRO test, the astronaut is asked to exert the MVC through a man-machine interface (MMI), and he is then asked to follow a force profile (presented as a line on the PC screen) at a fixed percentage of the MVC. Different and repeated sequences of visual feedbacks and/or proprioceptive feedback only (the PC screen becomes dark) were experienced by the astronaut during the experiment (see Materials and Methods). Baseline on ground data collections has also been performed pre- and postflight.

Analyzed parameters were divided in five main groups: power performance, cinematic, accuracy, muscular tremor, and proprioception. Each parameter analyzed monitors psychophysical and physiological factors most probably connected to CNS motion strategy, PNS organization, and muscular output level modification. In this study, we report that HGD and PFD performance degradation during long-term increments in space is not followed by adaptation, as reported during short-term increment experiments [8]. Once back on Earth, astronauts experienced recovery of upper limb performance.

\section{Materials and Methods}

2.1. Human Subjects. The work described in this manuscript has been carried out in accordance with The Code of Ethics of the World Medical Association (Declaration of Helsinki) for experiments involving humans. Informed consent was obtained for experimentation with human subjects. The CHIRO protocol was performed by 2 male astronauts.

2.2. Force Measurement. The HPA facility consists of two instruments: the HGD and PFD (Figure 1), which are isometric dynamometers designed and manufactured, respectively, for the best handgrip and pinch force application, with connection cables and terminal connectors to the interface box (IBOX). The IBOX is connected to power supply and to a laptop PC via a PCMCIA card, which is used both for dynamometer data acquisition and for real-time force level computation and visualization. The PC is used also as manmachine interface (MMI) and leads the test subject during the experiment execution. The HPA has also an instrumented glove with 15 degrees of freedom, allowing the study 
of the position of single phalanxes, coupled with an inertial platform with 6 degrees of freedom ( 3 accelerometers and 3 angular velocity sensors) placed on the wrist, allowing the execution of protocols on motion control strategies during grasping and reaching tasks and the study of alteration in cognitive processes.

HGD and PFD force ranges are, respectively, 40-1000 Newton and 0-270 Newton with a measurement accuracy of $0.75 \%$ of the full scale. Electronic signals from dynamometers are conditioned by the 6th order low pass Butterworth filter $\left(f_{\mathrm{L}}=20 \mathrm{~Hz}\right)$.

2.3. CHIRO Experimental Protocol. The Crew's Health: Investigation on Reduced Operability (CHIRO) protocol [8] consists of nine consecutive isometric force tests for each ground or flight session. Before each session, HPA software records subject's MVC and calculates three fixed force targets at $25 \%, 50 \%$, and $75 \%$ of MVC. At each target level, the astronaut is asked to perform three consecutive tests. Each test lasts 24 seconds and it is divided in three equally spaced time windows, respectively:

(1) Preblind visual feedback window (0-8 seconds)

(2) Only proprioceptive window (8-16 seconds)

(3) Postblind visual feedback window (16-24 seconds)

In the first window, the subject aims at the force target, and once reached, he tries to maintain the force output level. Both actions are supported by the visual feedback displayed on the PC monitor. In the second window, no visual feedback is given: the PC monitor is turned off and the subject uses only the proprioceptive information to maintain the force level output constant. In the last window, the visual feedback is restored on the PC monitor. At the end of the test, the complete 24-second time history is showed on screen to allow learning during rest time (12 seconds) prior to test repetition. The CHIRO protocol is applicable for both the HGD and PFD tools. The HGD protocol requires the tuning of the grip size according to the best comfort of the test subject. The PFD protocol might be executed with a pinch "key style" or in "finger opposition." The "key style" modality was used. In sessions performed during increments 7 and 8, the CHIRO protocol was performed by the astronauts after familiarization and training, as described below:

(1) Preflight ground sessions, called GROUND1 (G1) and GROUND2 (G2)

(2) Flight sessions, called FLIGHT1 (F1), FLIGHT2 (F2), and FLIGHT3 (F3)

(3) Postflight ground sessions, called GROUND3 (G3) and GROUND4 (G4)

During increment 7, two flight sessions were performed. The CHIRO sessions were performed following the timing hereby:

Increment 7 (180 days mission): (i) G1: performed 154 days before launch

(ii) G2: performed 86 days before launch

(iii) F1: performed 142 days postlaunch

(iv) F2: performed 170 days postlaunch

(v) G3: performed 8 days postlanding

(vi) G4: performed 16 days postlanding

Increment 8 (191 days mission):

(i) G1: performed 67 days before launch

(ii) G2: performed 29 days before launch

(iii) F1: performed 19 days postlaunch

(iv) F2: performed 88 days postlaunch

(v) F3: performed 150 days postlaunch

(vi) G3: performed 8 days postlanding

(vii) G4: performed 14 days postlanding

2.4. Data Analysis Method. We divided the analysis in five parameter's groups:

(1) Power parameters: including parameters involved with force performance directly exerted with HGD and PFD tools, like force output level and muscular fatigue interpreted as the subject's capacity to maintain force performance over time

(2) Accuracy in force modulation parameters: including parameters involved in constant force control of the muscular system and parameters correlated with subject's ability to maintain the force level near to the target as much as possible over time

(3) Muscular tremor parameters: including frequency analysis for the investigation of force modulation strategy and information about muscular tremors during isometric tests

(4) Cinematic parameters: applied to find a good parameter for gaining information about subject's ability to change the force level over time

(5) Proprioceptive parameters: the blind period of each test, where subject has no visual feedback

Each session contains nine consecutive tests for HGD and nine consecutive tests for PFD; global test duration (024 seconds) was divided in five-time subwindows with different features:

(1) $0-4$ sec: the first transient period where subject tries to reach the target in little time as possible and finds his force modulation equilibrium

(2) 4-8 sec: where subject tries to keep the force level constant by both proprioceptive and visual feedback 
MVC: Increment $8 \mathrm{HGD}$ and PFD

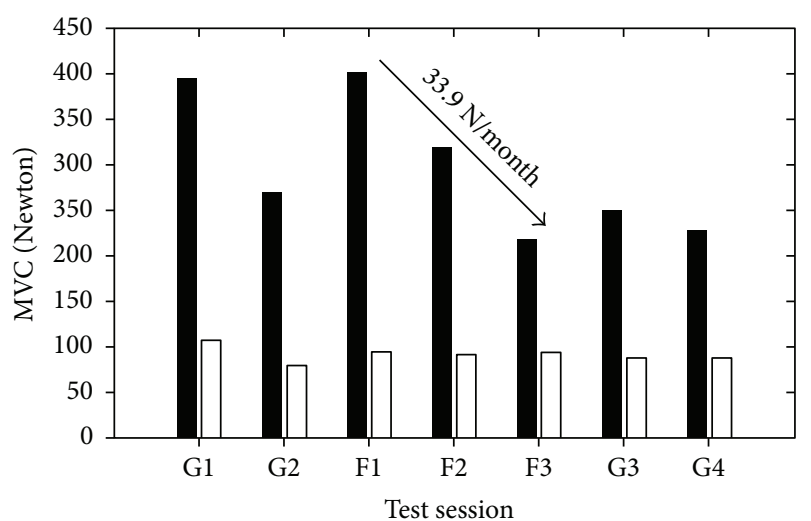

HGD

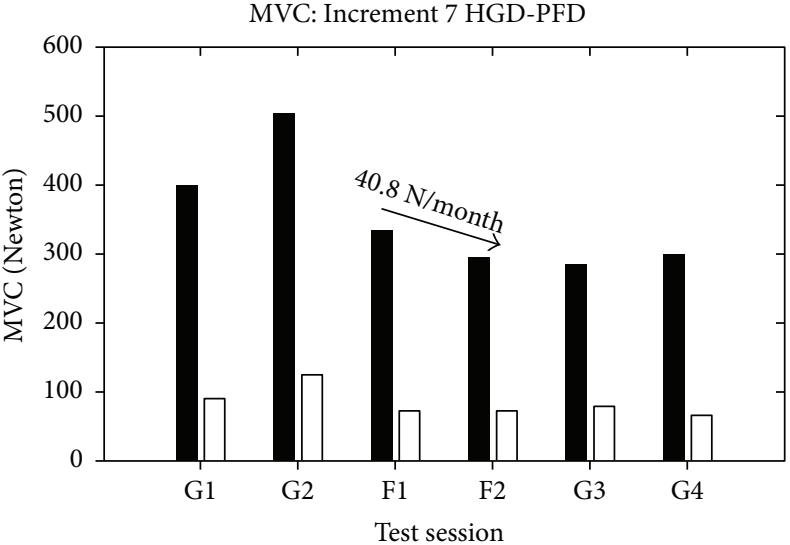

HGD PFD

(a)

(b)

Figure 2: Maximum voluntary contraction (MVC). (a) Trend for increment 8 mission. (b) Trend for increment 7 mission. The timing of the different sessions is reported in Materials and Methods.

(3) 8-16 sec: where subject tries to keep the force level constant by proprioceptive feedback only

(4) $16-17.5 \mathrm{sec}$ : the second transient period where subject recognizes the force level variation during the previous time window and tries to reach again the target after visual feedback is restored

(5) 17.5-24 sec: where subject performs the experiment with both proprioceptive and visual feedbacks, but with muscular fatigue predominance

Data were processed with Matlab software (MathWorks).

\section{Results}

3.1. Maximum Voluntary Contraction (MVC). The MVC is the maximum force value that a subject can exert constantly using HGD or PFD tools, for a time period of at least 3 seconds. MVC value is used also by the HPA software for the evaluation of the three target values $(25 \%, 50 \%$, and 75\% MVC). As shown in Figure 2(a), in test sessions during increment 8 mission, HGD-MVC values gradually decrease over time. A mean decrease of about $45 \%$ equivalent to 33.9 Newton/month was observed. PFD-MVC values do not show significant variation over time. Remarkably, a similar trend is reported for increment 7 mission as shown in Figure 2(b) (HGD-MVC values decrease of 40.8 Newton/month). During long-term missions, there is no adaptation to weightlessness and no recovery, and HGD-MVC values keep decreasing during weightlessness conditions. PFD-MVC values do not show gross variation over time as previously observed for short-term missions [8].

These data suggest that absence of recovery during long-duration (more than six months) space flights could potentially pose a serious risk for astronauts' health and performance of upper limbs due to the decrease over time of the MVC.
3.2. Static Effort (SE). The SE parameter allows the evaluation of the force deviation from target over time. Ideally, force output trace on the monitor must be equal to a rectangular function assuming that the subject reaches the target at $t=$ 0 and maintains the force level constant for 24 seconds. In practice, during the experiment, the subject does not reach the target in the time window $0-4$ seconds. The muscular system response might be represented with a second order Hill's model [9]. The SE parameter refers to the difference between the area under real curve (AURC) and the area under ideal curve (AUIC), described by the following equations:

$$
\begin{aligned}
& \mathrm{SE}=1-\frac{\mathrm{AURC}}{\mathrm{AUIC}}, \\
& \mathrm{SE}_{i}=1-\frac{\mathrm{AURC}_{4-24}}{T_{i} \cdot 20},
\end{aligned}
$$

where SE is the static effort; AURC is the area under real curve; AUIC is the area under ideal curve; $\mathrm{AURC}_{4-24}$ is calculated in the time window 4-24 seconds excluding the initial transient period $0-4$ seconds. $T_{i}$ is the target value; $i$-index represents the test number.

If no force is applied, the SE value is equal to 1 . When force is applied, $\mathrm{SE}$ values $0<\mathrm{SE} \leq 1$ are expected. In weightlessness, SE shows larger values for PFD tests rather than HGD. Particularly, 4-24 seconds gray bars highlight that adaptation does not occur during long-term space missions, as reported in Figure 3(a) and Figure 3(b). These data could be explained assuming that during PFD tests, small mass muscles are involved both in force generation and modulation, thus resulting in a higher fatigue for the small muscles. Conversely, during the short-term experiment [8], data from the SE tests showed that the subject develops a mechanism of adaptation to weightlessness involving the compensation of a bigger muscle fatigue with increase in force modulation, therefore adapting the upper limbs to the microgravity environment. Hence, a primary difference between short-term 


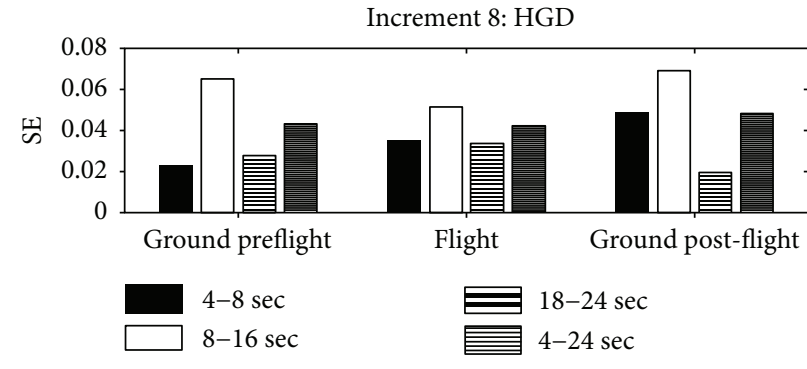

(a)

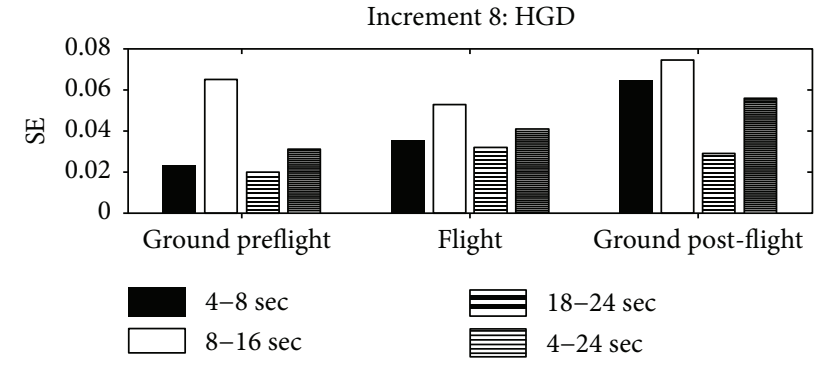

(b)

Figure 3: Static effort (SE). (a) Trend for increment 8 in handgrip dynamometer (HGD) tests. (b) Trend for increment 8 in pinch force dynamometer (PFD) tests. Average values of preflight, flight, and postflight are reported.

and long-term missions is that astronauts' upper limbs do not show adaptation (as shown by SE measurements).

3.3. Mean Distance from Target (MDT). Considering the output force trend, the MDT parameter is used to investigate subject's ability to keep the output force level near to the target during the global test duration (24 seconds). MDT is calculated as follows:

$$
\mathrm{MDT}_{i}=\frac{1}{N} \cdot \sum_{i=1}^{N}\left|F_{i}-T\right|,
$$

where MDT is the mean distance from the target. $N$ is the number of data sampled for each test. $F_{i}$ is the force output level measured by HGD or PFD tools. $T$ is the target value.

The mean value of MDT calculated for each test group for the same target value is shown in Figure 4. HGD tests show a decrease of $48.4 \%$ of power during consecutive flight sessions while PFD tests do not show any gross variation. This finding can be explained by the decrease of HGDMVC, which could potentially determine a smaller reaction capacity in big mass muscles. PFD-MDT showed no significant variation, accordingly with PFD-MVC parameter.

3.4. Force Variance $(F V)$. Force variance evaluation is a proper method to investigate subject's ability to keep the output force level constant. It is independent from target level, and it was performed during each time window $4-8$ seconds, $8-16$ seconds, and $18-24$ seconds. In statistics, variance is defined as a data dispersion index as follows:

$$
\mathrm{FV}=\sigma^{2}=\frac{1}{n} \sum_{i=1}^{n}\left(X_{i}-\mu\right)^{2}
$$

where FV is the force variance. $n$ : number of considered elements. $\mu$ is the average force data values of vector $\mathbf{X}$.

Variance is zero only if all force data are equal to each other and therefore only if all data are equal to the data average, but it increases with the increase of data difference. In general, $\mu$ is lower than force target. Therefore, using variance as evaluation parameter allows the estimation and quantification of force fluctuation. Moreover, variance is a complementary parameter for data analysis because it allows the consideration of the subject's chance of not reaching

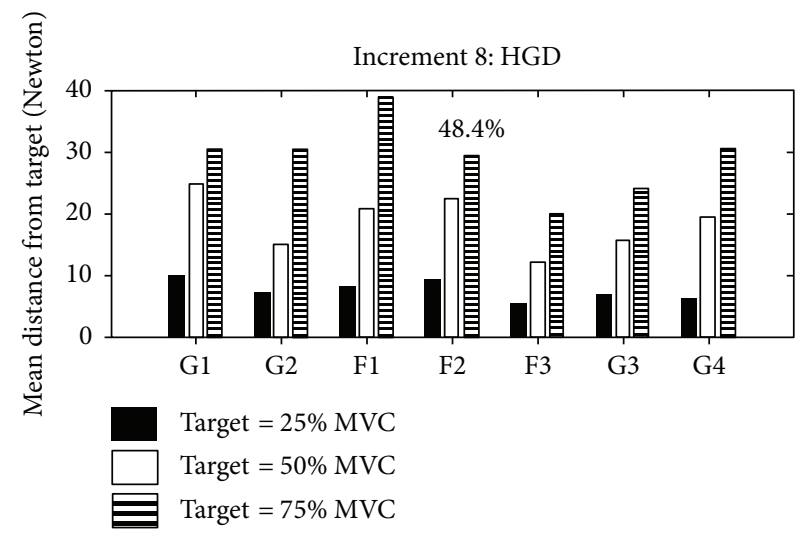

(a)

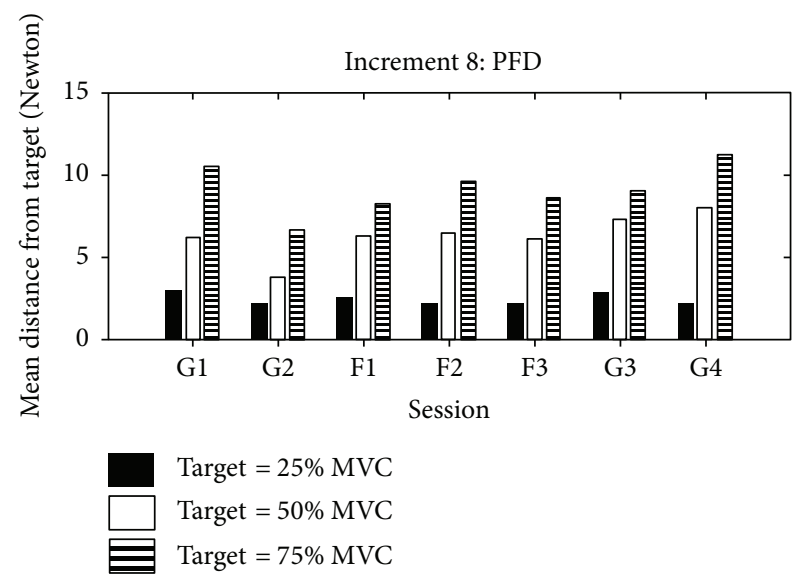

(b)

Figure 4: Mean distance from target (MDT). (a) Analysis for handgrip dynamometer (HGD) tests. (b) Analysis for pinch force dynamometer (PFD) tests. The timing of the different sessions is reported in Materials and Methods.

exactly the target level, maintaining the force level inside the tolerance area visualized on the PC monitor by upper and lower horizontal lines, and keeping his personal force level (generally different from target level) constant. Figure 5 shows the variance parameter for mission increment 8 relatively to HGD and PFD tests, for the considered time windows. Three data groups are reported as the mean value of variance for ground (preflight), flight, and ground (postflight) sessions. 


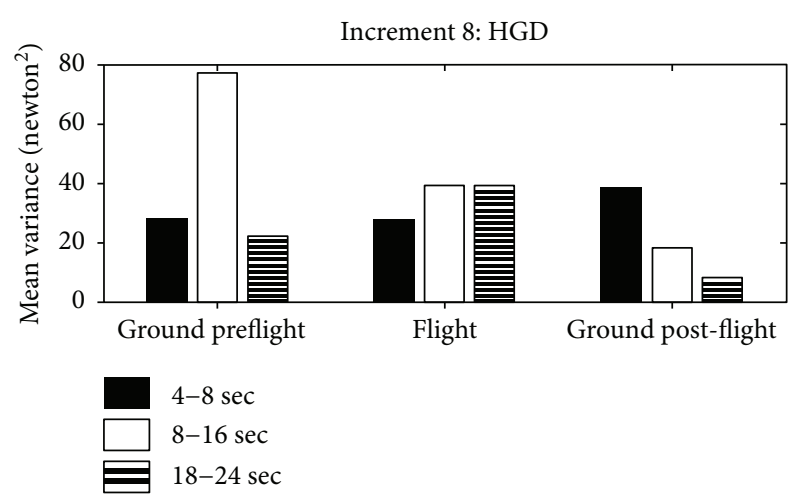

(a)

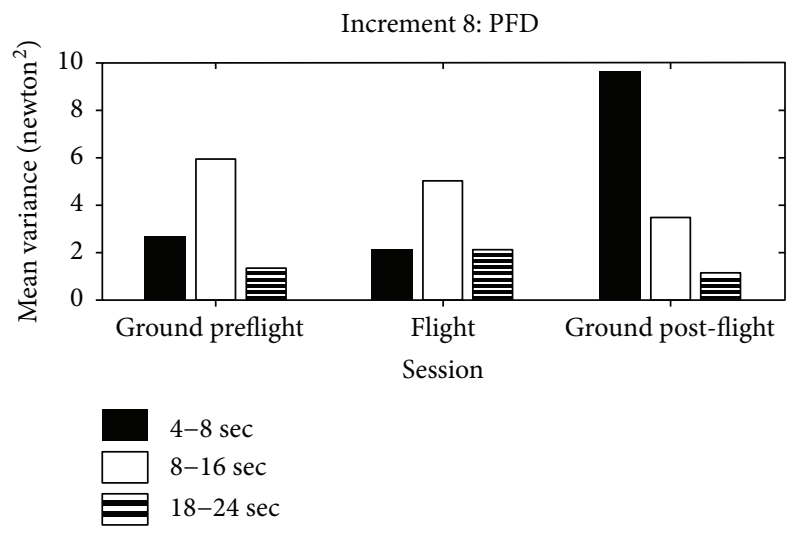

(b)

FIGURE 5: Force variance (FV). (a) FV parameter analysis for handgrip dynamometer (HGD) tests. (b) FV parameter analysis for pinch force dynamometer (PFD) tests.

Two different data trends can be assessed for HGD and PFD tests: variance decreases in weightlessness conditions in 4-8 seconds and in the only proprioceptive window (8-16 seconds), while it increases in the 18-24-second time window. This effect might be explained, at least partially, by the muscular fatigue appearance during the last eight seconds of each test, which nullifies the subject's attempt to keep his force level constant. Variance trend correlates with the fact that muscular fatigue is more evident in microgravity rather than $1 \mathrm{~g}$ Earth gravity.

3.5. Maximum Force Range (DELTA). The DELTA parameter was used to investigate the subject's force range for modulation and accuracy evaluation, defined by the following equation:

$$
\text { DELTA }=|F \max -F \min |
$$

Figure 6(a) shows a representative graph from a test DELTA for HGD, in the three time windows: 4-8 seconds, 8-16 seconds, and 18-24 seconds, calculated, respectively, for ground (preflight), flight, and ground (postflight) sessions. DELTA decrease in weightlessness conditions is reported in the proprioceptive time window (8-16 seconds, Figures 6(a) and 6(b)).
3.6. Force Tremor (FT). A frequency analysis of force data with HGD and PFD was performed using the power spectral density (PSD) of each signal. We applied the WienerKintchine theorem that allows the PSD calculation by fast Fourier transform (FFT) of the autocorrelation function. The sampling rate of the HPA hardware was fixed at $100 \mathrm{~Hz}$, with a Butterworth 6th order low pass filter in the frequency window $0-20 \mathrm{~Hz}$ including detection for the 8$12 \mathrm{~Hz}$ tremor effects [10].

During contractions, hand muscle movements are characterized by physiological finger tremor [11] induced by muscular fiber recruitment. This physiological effect is amplified by isometric tests when a subject tries to keep the force level constant [12]. The FFT module increases as the target level increases, and the PSD is concentrated in the power spectral band $0-3.5 \mathrm{~Hz}$ [13] both for HGD and PFD tests, as shown in Figure 7(a). With respect to the $4-20 \mathrm{~Hz}$ spectral band, Figures 7(b) and 7(c) show the presence of small frequency components versus the FV parameter described in the previous paragraph.

During the test sessions performed, the PSD behavior reported in Figures $7(\mathrm{~b})$ and 7 (c) shows common features of the presence of small frequency components in the 4$20 \mathrm{~Hz}$ band especially at lower FV values. The PSD parameter is a proper mean to investigate subject force modulation strategy. In the attempt of maintaining a constant force level, big mass muscles are modulated in the frequency range of 0 $3.5 \mathrm{~Hz}$ and are responsible for mean force level. Conversely, small mass muscles and finger's muscles are modulated at higher frequency $(4-20 \mathrm{~Hz})$ for the regulation of fine force levels and big muscle fluctuations. No gross differences were observed between ground and flight PSD sessions, confirming that PSD is not gravity dependent as already reported in precedent studies $[11,12]$.

3.7. Contraction Speed (CS). HGD and PFD are isometric tools used if muscular length variation is not evident. Conversely, the CS parameter allows for the evaluation of muscle capacity in force variation in the time unit considered, as described by the following equation:

$$
\mathrm{CS}_{i}=\frac{\Delta F_{i}}{\Delta T}=\frac{F_{i+1}-F_{i}}{\Delta T}(\text { Newton } / \mathrm{sec})
$$

where CS is the contraction speed. $F_{i}$ is the force output level measured by HGD or PFD. $\Delta T$ is the sampling time (10 msec).

Stressed or not, efficient muscles are not able to perform high level of CS. With respect to the CHIRO protocol, this parameter has been evaluated in the time windows $0-4 \mathrm{sec}-$ onds and $0-24$ seconds. At test beginning $(t=0)$, subject's intention is to reach the target force as fast as possible, and thus the CS parameter is used to investigate the impulsive response of the muscular system in the time window $0-4 \mathrm{sec}-$ onds. When the subject switches from proprioceptive to visual feedback, the analysis of force data CS allows for investigation of subject's reaction capacity. As shown in Figures 8(a) and 8(b), during long-term flight, the impulsive response (at $75 \%, 50 \%$, and $25 \%$ MVC) and reaction capacity (at 75\%, 50\% MVC) for HGD test decrease 


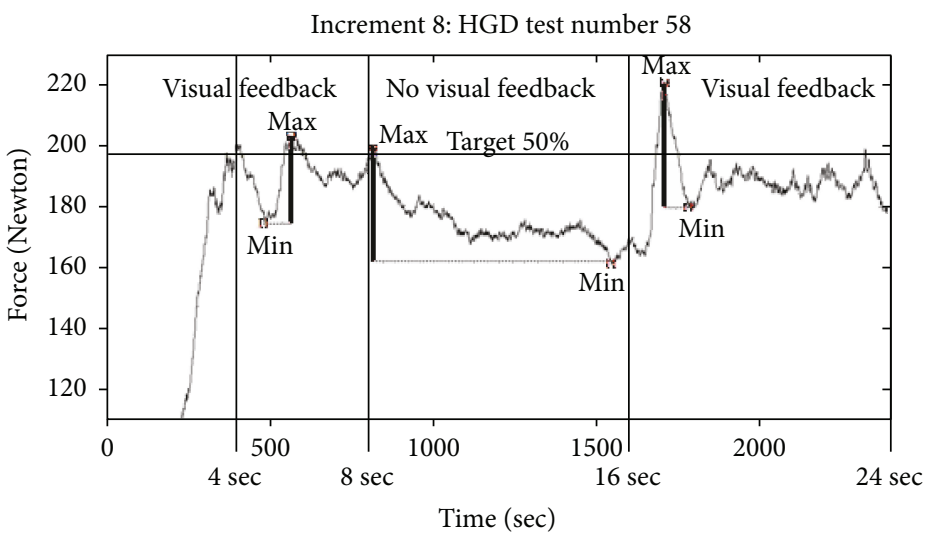

(a)

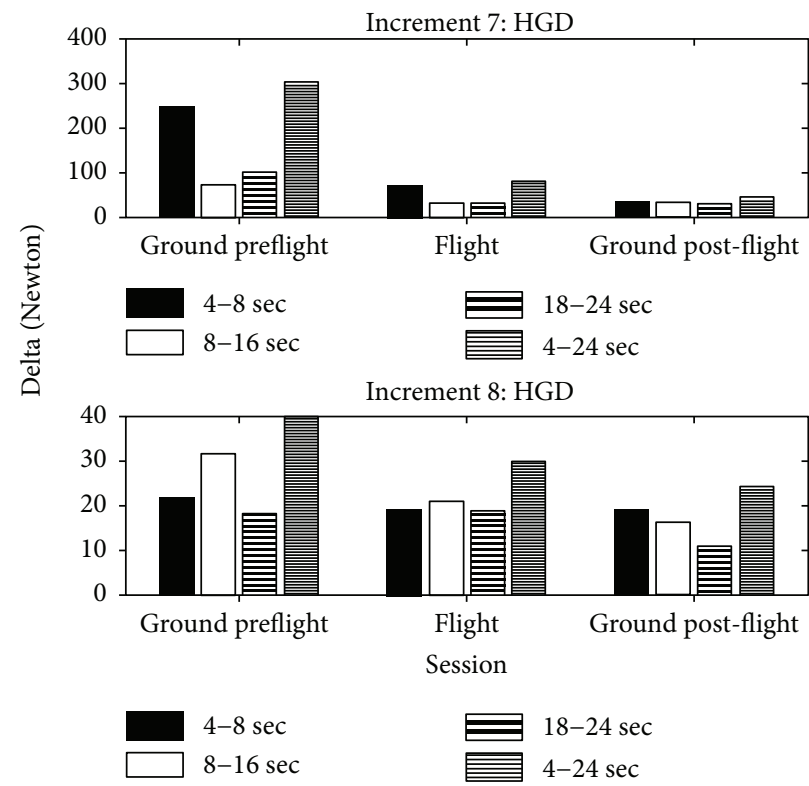

(b)

Figure 6: Maximum force range. (a) DELTA definition for each time window. (b) DELTA trend for handgrip dynamometer (HGD) tests.

strikingly (45\% of CS-FLIGHT), while the CS for PFD is not affected (data not shown), as for MVC. Therefore, during long-term missions, MVC and CS are dependent on each other: MVC shows no adaptation over time while CS values decrease continuously in microgravity.

3.8. Proprioceptive Parameters. In the time window 8-16 seconds, the visual feedback is lost, and thus the astronaut relies only on the proprioceptive feedback during his attempt to keep constant the force level. A parameter called target distance (TD) was used to investigate the subject ability to keep the force level constantly near the target level during visual feedback absence. TD is calculated by the following equation:

$$
\mathrm{TD}_{i}=\left|F_{i=16 \mathrm{sec}}-T\right|
$$

where $\mathrm{TD}$ is the force-distance value from the target. $F_{i=16 \mathrm{sec}}$ is the force measured at $T=16 \mathrm{sec}$. $T$ is the force level target.
Intriguingly, as shown in Figures 9(a) and 9(b), TD clearly decreases in weightlessness conditions both for HGD and PFD tests, that is, astronauts perform this task better in weightlessness than on Earth. These data could be explained by the fact that during microgravity, the muscular proprioceptive feedback needs only to evaluate the force applied to the dynamometer rather than evaluating also the gravity force, present on Earth.

\section{Discussion}

In the present work, we found that during two long-term missions, the performance of the upper limbs measured by using HGD and PFD devices in space is not followed by adaptation to the space environment as reported during short-term missions [8]. Consequences of these findings are predictable: a dramatic impact on the strength of the astronaut's upper limbs engaged in long-term missions (>6 months) needs to be taken into account. Our results are in accordance with previous NASA studies that demonstrate 


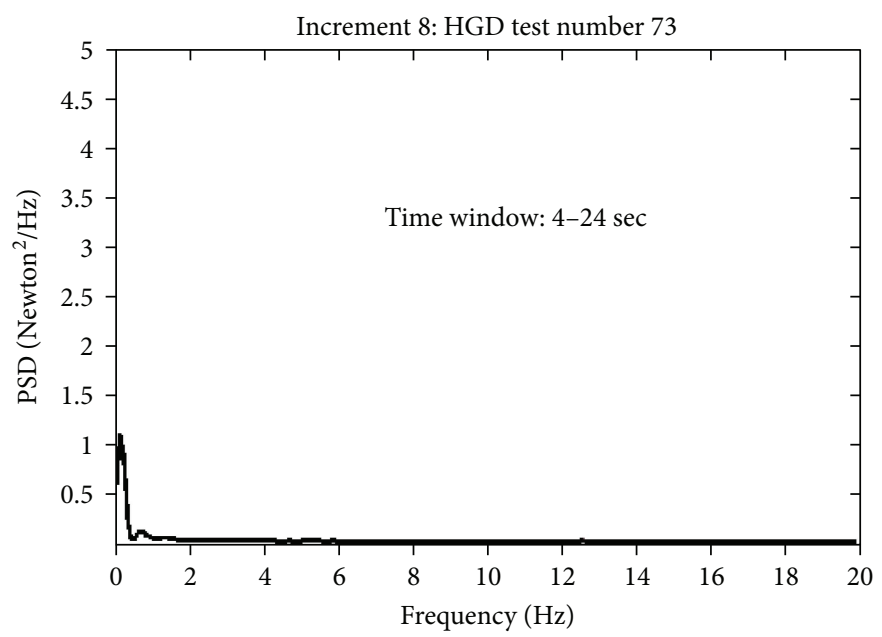

(a)

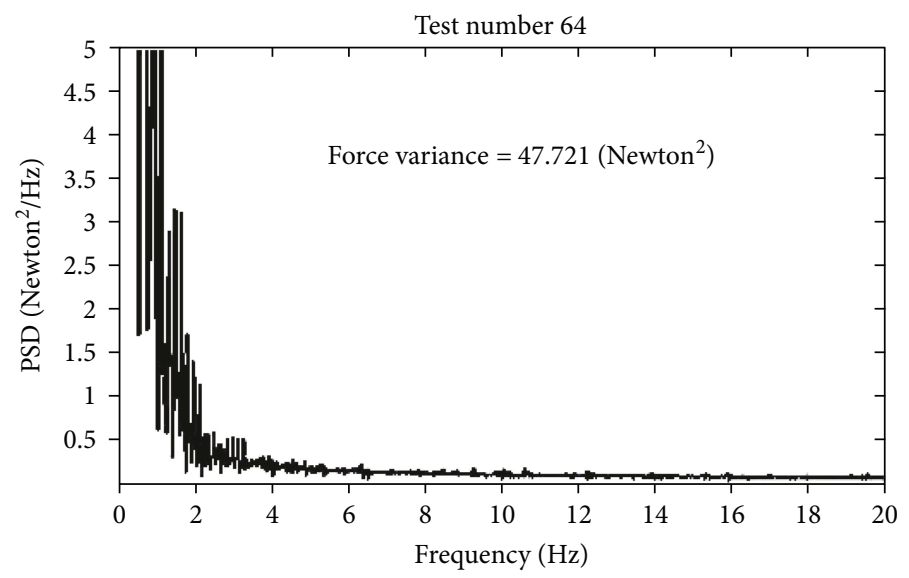

(b)

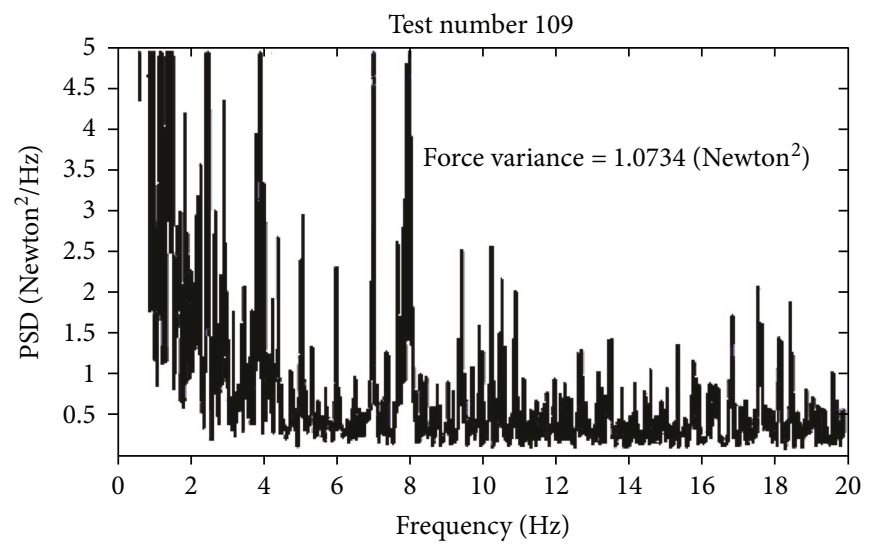

(c)

Figure 7: HGD power spectral density (PSD). (a) HGD-PSD for 0-20 Hz frequency window. (b) Test session 64. HGD-PSD 4-20 Hz frequency window. (c) Test session 109. HGD power spectral density (PSD) $4-20 \mathrm{~Hz}$ frequency window. Data acquired at $25 \%$ MVC.

that even bones are affected over the course of long-term missions by loss of their total hip bone mass [14]. Moreover, our data add information to the list of known effects of space flights on the human body during long-duration missions such as muscle atrophy, bone demineralization, altered immune function $[15,16]$, cardiovascular system dysfunction $[17,18]$, and also alterations in sleep function [19]. A significant amount of data on the decrease in the performance of various groups of muscles has been collected from astronauts flying from 1989 to 1995 in NASA's orbiters or Russian capsules [20]. The results from these investigations were expected to provide evidence to support a program of preflight conditioning, in-flight countermeasures, and postflight rehabilitation protocols. In general, the decrease in 


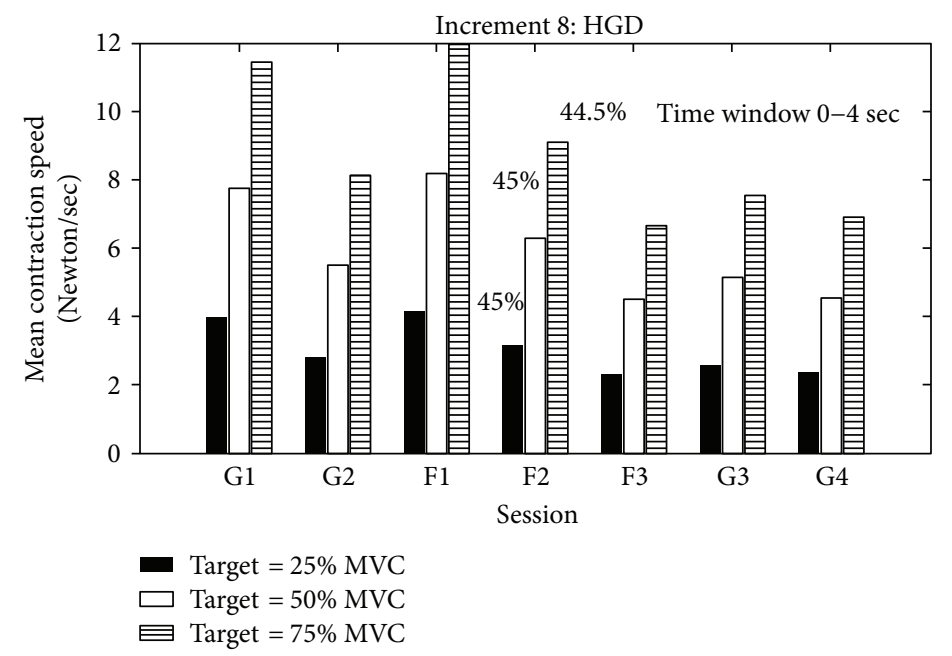

(a)

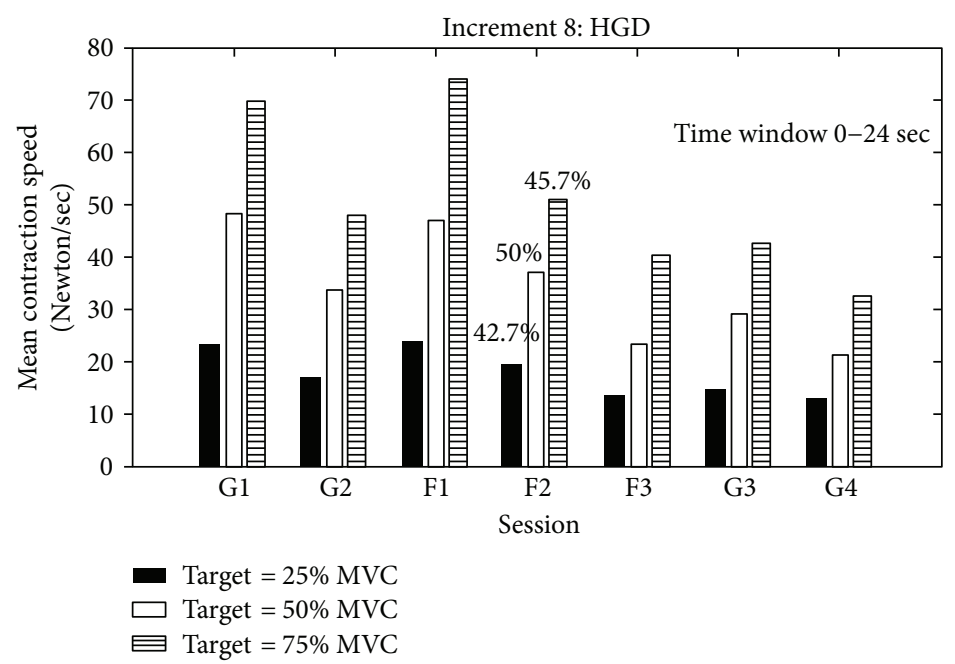

(b)

Figure 8: Contraction speed. (a) Handgrip dynamometer- (HGD-) impulsive response in the time window 0-4 seconds. The values of the mean contraction speed decrease, respectively, from F1 to F3 of $45 \%$ for $25 \%$ and $50 \%$ and $44.55 \%$ for $75 \%$ MVC tests. (b) Handgrip dynamometer- (HGD-) contraction speed (CS) in the time window 0-24 seconds. The values of the mean contraction speed decrease, respectively, from $\mathrm{F} 1$ to $\mathrm{F} 3$ of $42.7 \%$ for $25 \%, 50 \%$ for $50 \%$, and $45.7 \%$ for $75 \%$ MVC tests. The timing of the different sessions is reported in Materials and Methods.

muscle strength and volume was systematic, affecting many major muscle groups analyzed.

In our study, we found that in general HGD tests show a lower performance than PFD. Muscular microfilament performance could be reduced by the decrease in calcium concentration or by other physiological factors correlated with calcium metabolism [21]. In weightlessness conditions, the control of the force level and the maintenance of constant force level improve; however, for higher target level (75\% of MVC), the muscular fatigue effects are evident during the course of the experiment. Force modulation and muscular tremor effects could be subject dependent. A subject could show better constant force output performance using small muscles modulated at higher frequency $(0$ to $4 \mathrm{~Hz}$, frequency for principal big mass muscles and 4 to $20 \mathrm{~Hz}$ for small mass muscles). This strategy seems to be good for $25 \%$ and $50 \%$ of
MVC force level but it is not efficient for $75 \%$ of MVC force level, probably because small mass muscle effects are completely masked by high force produced by big mass muscles. Finally, proprioceptive feedback appears to perform better in weightlessness conditions. Of interest, during the flight session 1 (F1) that was performed 19 days postlaunch, we were able to confirm the results from Pastacaldi et al. [8] that analyzed the MVC and PFD of astronauts during short-term exposure to microgravity.

Once back on Earth, astronauts experience recovery of upper limb performance. We speculate that the lack of visual feedback and gravity force input signal would make the CNS more effective in performing the requested tasks.

On Earth, the CHIRO protocol has been applied to healthy women volunteers to evaluate changes of muscle strength on the upper dominant limb with respect to age 


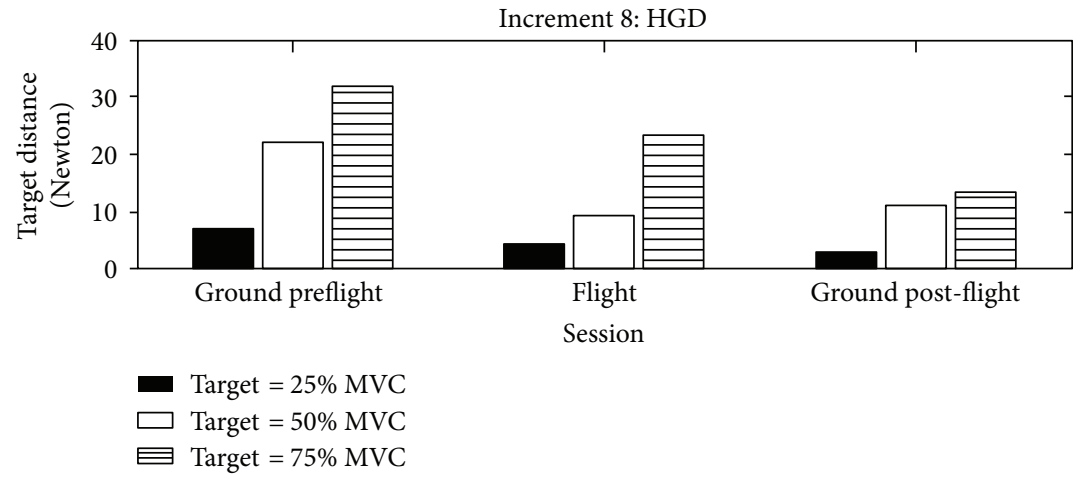

(a)

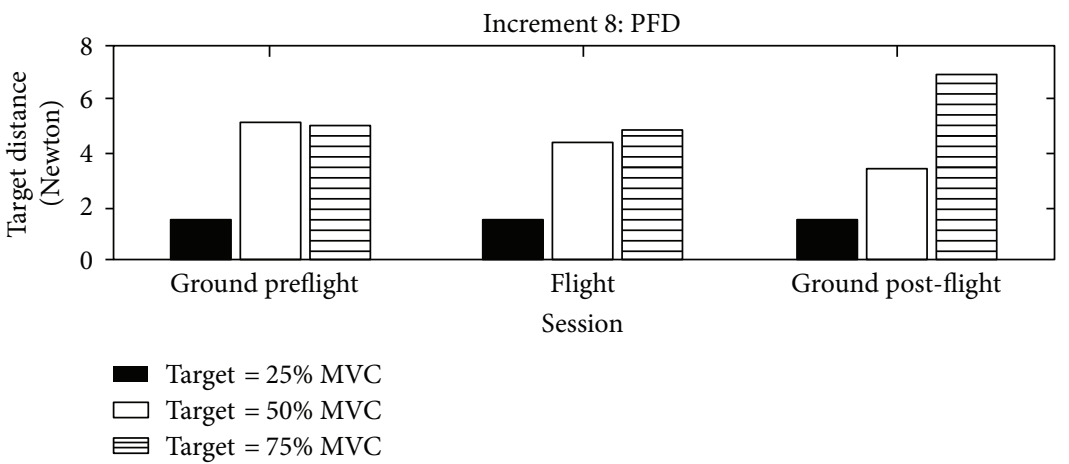

(b)

Figure 9: Proprioceptive parameters. (a) Handgrip dynamometer (HGD) target distance (TD). (b) Pinch force dynamometer (PFD) target distance (TD).

and menopausal status [22]. The investigators found that handgrip strength was strongly associated with age: muscle strength is progressively decreased in the volunteers older than 40 years old. A comparison between the data collected in space and on Earth would provide further understanding and might help to indicate possible countermeasures both for astronauts involved in mission longer than six months and for patients affected by muscular disorders or elder people on Earth. In this context, the HPA device could be adopted as a valuable tool to investigate upper limb strength and performance over time in a variety of research or clinical settings.

\section{Abbreviations}

MVC: Maximum voluntary contraction

HPA: Hand posture analyzer

HGD: Handgrip dynamometer

CNS: Central nervous system

PNS: $\quad$ Peripheral nervous system

PFD: $\quad$ Pinch force dynamometer

CHIRO: Crew's Health Investigation on Reduced Operability

IBOX: Interface box

MMI: Man-machine interface

ISS: International Space Station

ESA: $\quad$ European Space Agency

ASI: $\quad$ Italian Space Agency
NASA: National Aeronautics and Space Administration

EVA: Extravehicular activities.

\section{Conflicts of Interest}

The authors declare that they have no conflicts of interest.

\section{Acknowledgments}

This work has been supported by the Italian Space Agency (ASI) in the frame of the ASI-NASA Memorandum of Understanding (MOU) for the utilization of the International Space Station (ISS). The authors acknowledge Professor Paolo Pastacaldi for his initial contribution to the project.

\section{References}

[1] T. Lang, J. J. W. A. Van Loon, S. Bloom et al., "Towards human exploration of space : the THESEUS review series on muscle and bone research priorities," npj Microgravity, vol. 3, no. 1, 2017.

[2] N. Petersen, P. Jaekel, A. Rosenberger et al., "Exercise in space : the European Space Agency approach to in-flight exercise countermeasures for long-duration missions on ISS," Extreme Physiology \& Medicine, vol. 5, no. 1, pp. 1-13, 2016.

[3] S. Appendino, E. P. Ambrosio, F. C. Chen et al., "Effects of EVA glove on hand performance," in 41st International Conference on Environmental Systems, pp. 17-21, Portland, Oregon, USA, July 2011. 
[4] A. Carey and S. K. Robinson, "An unpowered exoskeleton to reduce astronaut hand fatigue during microgravity extra vehicular activity (EVA)," in AIAA SPACE 2016, Long Beach, California, USA, September 2016.

[5] V. Zolesi, L. Serafini, S. Baldacci, G. Neri, L. Liuni, F. Flore et al., "New protocols for the analysis of the performances of the human upper limb on the international space station," in AIAA Space 2003 Conference \& Exposition, pp. 23-25, Long Beach, CA, USA, September 2003.

[6] S. Baldacci, G. Neri, V. Zolesi, and V. Cotronei, "Hand posture analyzer (HPA): portable instruments for upper limb posture analysis," 2001 Conference and Exhibit on International Space Station Utilization, 2001, pp. 15-18, Cape Canaveral, Florida, USA, October 2001.

[7] B. D. Lawson and A. M. Mead, "The sopite syndrome revisited: drowsiness and mood changes during real or apparent motion," Acta Astronautica, vol. 43, no. 3-6, pp. 181-192, 1998.

[8] P. Pastacaldi, P. Orsini, F. Bracciaferri et al., "Short term microgravity effect on isometric hand grip and precision pinch force with visual and proprioceptive feedback," Advances in Space Research, vol. 33, no. 8no. 8, pp. 1368-1374, 2004.

[9] A. V. Hill, "The heat of shortening and the dynamic constants of muscle," Proceedings of the Royal Society B: Biological Sciences, vol. 126, no. 843, pp. 136-195, 1938.

[10] R. J. Elble and J. E. Randall, "Motor-unit activity responsible for 8 - to $12-\mathrm{Hz}$ component of human physiological finger tremor," Journal of Neurophysiology, vol. 39, no. 2, pp. 370383, 1976.

[11] E. Gallasch, M. Moser, I. B. Kozlovskaya, T. Kenner, and A. Noordergraaf, "Effects of an eight-day space flight on microvibration and physiological tremor," American Journal of Physiology-Regulatory, Integrative and Comparative Physiology, vol. 273, no. 1, pp. R86-R92, 1997.

[12] E. Gallasch and I. B. Kozlovskaya, "Vibrografic signs of autonomous muscle tone studied in long term space missions," Acta Astronautica, vol. 43, no. 3-6, pp. 101-106, 1998.

[13] A. B. Slifkin, D. E. Vaillancourt, and K. M. Newell, "Intermittency in the control of continuous force production," Journal of Neurophysiology, vol. 84, no. 4, pp. 1708-1718, 2000.

[14] "How long does it take to rebuild bone lost during space flight?," http://www.nasa.gov/mission_pages/station/research/ subregional_bone.html.

[15] J. Vernikos, "Human physiology in space," BioEssays, vol. 18, no. 12, pp. 1029-1037, 1996.

[16] B. Crucian, R. P. Stowe, S. Mehta, H. Quiriarte, D. Pierson, and C. Sams, "Alterations in adaptive immunity persist during long-duration spaceflight," npj Microgravity, vol. 1, no. 1, pp. 1-10, 2015.

[17] K. Otsuka, G. Cornelissen, Y. Kubo et al., "Intrinsic cardiovascular autonomic regulatory system of astronauts exposed long-term to microgravity in space : observational study," npj Microgravity, vol. 1, no. 1, pp. 1-8, 2015.

[18] R. L. Hughson, J. K. Shoemaker, A. P. Blaber et al., "Cardiovascular regulation during long-duration spaceflights to the International Space Station," Journal of Applied Physiology, vol. 112, no. 5, pp. 719-727, 2012.

[19] E. E. Flynn-evans, L. K. Barger, A. A. Kubey, J. P. Sullivan, and C. A. Czeisler, "Circadian misalignment affects sleep and medication use before and during spaceflight," npj Microgravity, vol. 2, no. 1, pp. 1-6, 2016.
[20] M. C. Greenisen, J. C. Hayes, S. F. Siconolfi, and A. D. Moore, Extended Duration Orbiter Medical Project Final Report, 1999.

[21] S. M. Smith, T. McCoy, D. Gazda, J. L. L. Morgan, M. Heer, and S. R. Zwart, "Space flight calcium: implications for astronaut health, spacecraft operations, and earth," Nutrients, vol. 4, no. 12, pp. 2047-2068, 2012.

[22] C. Cipriani, E. Romagnoli, V. Carnevale et al., "Muscle strength and bone in healthy women: effect of age and gonadal status," Hormones, vol. 11, no. 3, pp. 325-332, 2012. 


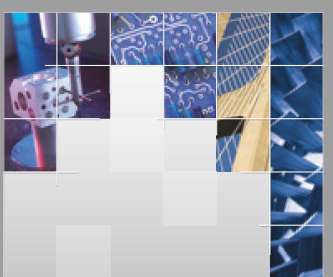

\section{Enfincering}
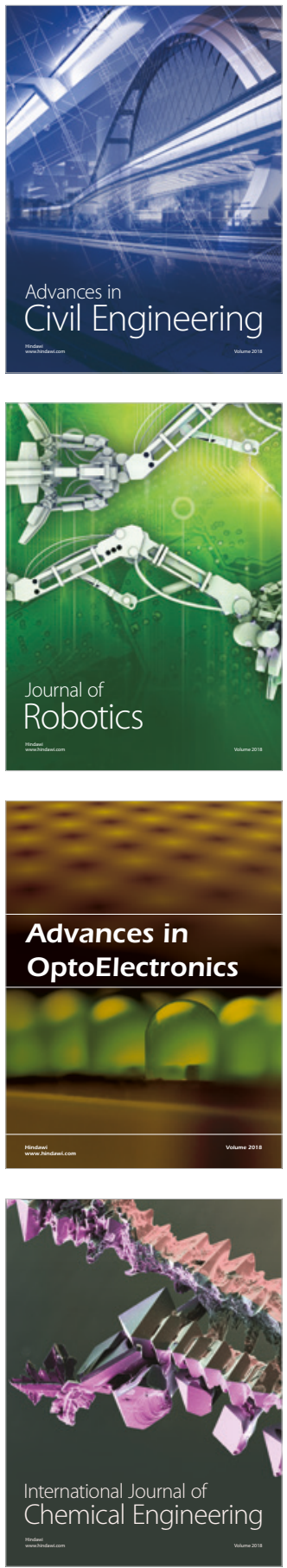

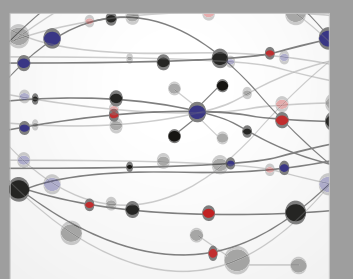

\section{Rotating \\ Machinery}

The Scientific World Journal

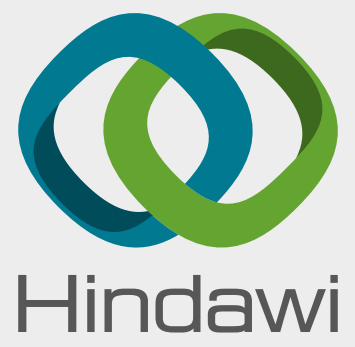

Submit your manuscripts at

www.hindawi.com
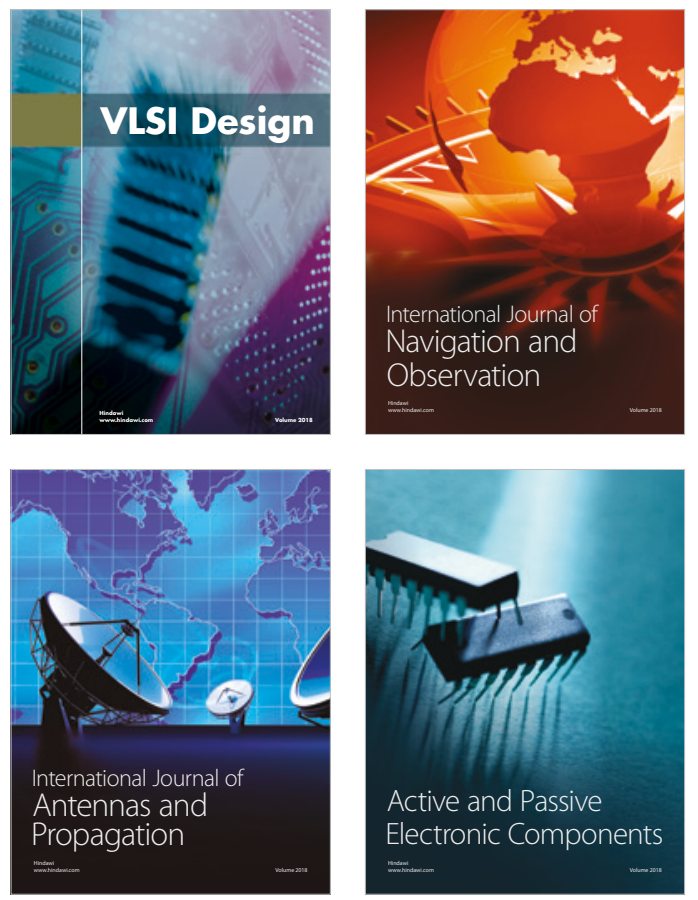
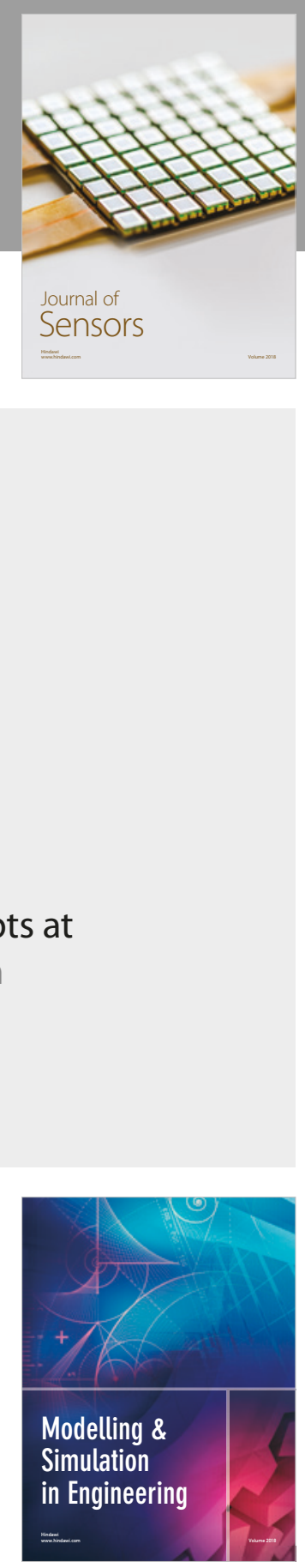

\section{Advances \\ Multimedia}
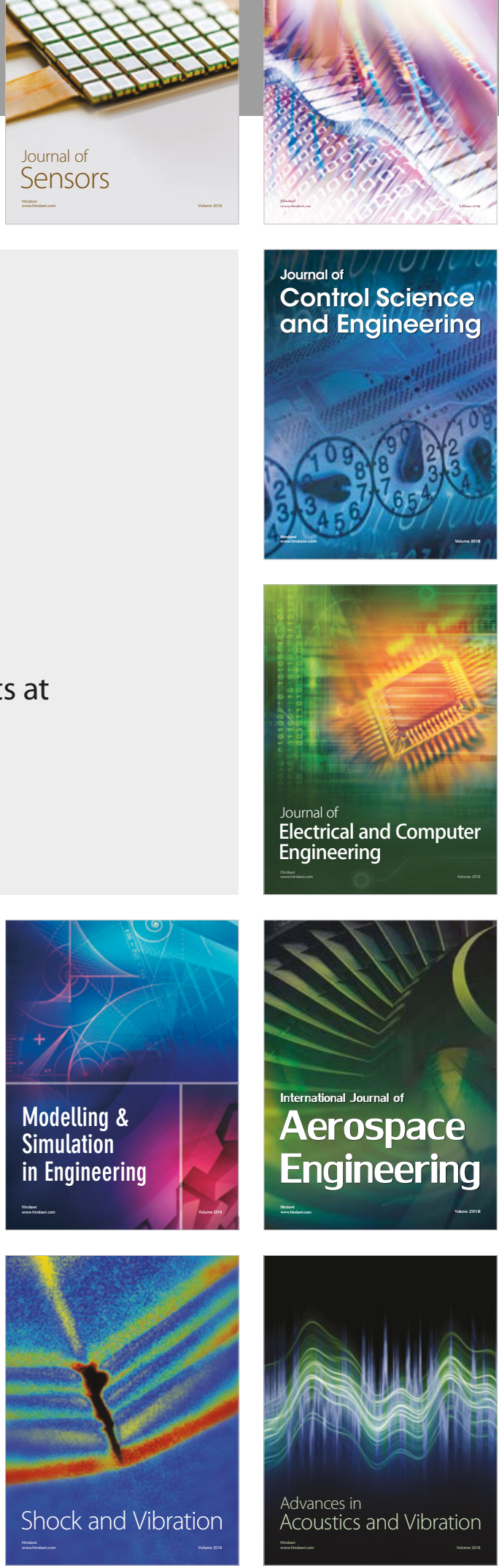